\title{
Animation Trends in Education
}

\author{
Lirong Xiao
}

\begin{abstract}
In the paper, we give a survey of animation content in education. At present, there is an extensive literature addressing the impact of animation in education and psychology fields. However, in animation field, although some software companies have developed their individual production toolboxes or platforms for animation content in education, there is lack of relevant research from the perspective of animation techniques. This paper first gives a survey of current animation content in education and then presents some potential trends of animation content in teaching.
\end{abstract}

Index Terms-Animation content, animation trends.

\section{INTRODUCTION}

Animation is playing a more and more important role in the classroom with the advent of computers. Lots of content have been developed for various disciplines or professional trainings. From picture galleries to complicated numerical simulations, animation provides us a complementary learning experience. The traditional education methods usually lacked effective approaches of illustrating an intuitional and clear content, while animation can compensate through the use of new software and hardware techniques. The most common animation content is developed by using either Flash or Java based technologies. From the perspective of animation application, there is more room in teaching in the classroom compared to other learning approaches. Thus, we focus on the animation content in the classroom in this survey.

The animation content in the classroom can be classified in three main types:

- Expositive: the users watch the expository content on screens;

- Interactive: the users can interact with the content at a higher level;

- Quizzes: the users are tested on a specific content.

Most of the content is developed in 2D with some of it developed by $3 \mathrm{D}$ technology. However, the content is displayed on 2D screens no matter the technology used to develop it. Nowadays, with the advent of new techniques, teaching has a natural need to move itself to 3D full experience. This means that the user can be immersed in a 3D environment and interact with the objects. The higher level of immersiveness comes from the fact that a very precise simulation of the real world is realized.

There is an extensive literature that addresses the impact of animation in education and psychology fields. However, from the perspective of animation techniques, there is lack of

Manuscript received November 22, 2012; revised March 18, 2013.

Lirong Xiao is with the Guangzhou Overseas Chinese Foreign Language School, No.1 Youai road, Huan Shi East road, Overseas New Village, Guangzhou, China (e-mail: xiao200912@gmail.com). research on the animation content in education. The purpose of this paper is to first survey the current developments and then further give the potential trends in teaching.

\section{StATE OF THE ART}

Animation in the classroom is becoming more and more popular in education. From teachers to students, almost all people have the experience of using animation in PowerPoint. Many people even have the experience of using some professional software package, e.g. maya. In terms of the above-mentioned classification, we will review the expository animation and the interactive animation respectively in this section.

\section{A. Expository Animation}

The expository animation content usually refers to the use of the animated video. Users use some specified software toolboxes to produce their individual cartoon videos, illustrating a topic in the classroom. Some software companies have developed various production tools and resources for almost all disciplines or various professional trainings. For example, GoAnimate.com claims that its educational division have provided 2.500 schools with its animation tools since December of 2010 [1]. Xtranormal.com claims that its registered users have jumped from 800,000 to 2.4 million in the last six months across disciplines and industries [2]. In addition to Twitter, blogs and YouTube videos, there are lots of the expository animation based tutorials to convey complex topics in the classroom.

There have been many educational researches on the effectiveness of expository animation in the classroom [3]-[5]. They have compared static and animated displays across a number of different content areas. Roughly speaking, using animations in the classroom are not intrinsically more effective than traditional static graphics. The key point is how to demonstrate the particular characteristics of individual animations or how to organize the teaching content in a specific lesson. For some students, the animated video of a complex topic may be beyond the limit of their learning capacity. Pausing the video and adding a writing or face-to-face explanation can circumvent this problem. If the learners can control the speed of the video, this can also help them get the maximum amount of benefit from this technique.

In general, based on the production tools provided by Xtranormal.com, GoAnimate.com and Animasher.com, producing an expository animation video follows the steps below.

- Pick a character. Users customize their individual characters with features like a potbelly, cat-eye glasses, a bouffant hairdo or gorilla hands; 
- Pick a background. Animasher's options [6] include a lecture hall, a swimming pool and an exploding atom bomb;

- Add dialogue. Users input in their own dialogue and select accent. Production tools can generate the synthesis voice accordingly. Animasher offers sound effects like fireworks and screams as background sound;

- Direct. Users can edit the scene, e.g. to add pauses, motions, to adjust camera angles, even cut and zoom.

\section{B. Interactive 3D Animation}

In education, new media technologies and forms provide more options and opportunities to improve the productivity of learning. It is straightforward to integrate the latest techniques of real-time character animation, 3D visualizations and $3 \mathrm{D}$ simulations into other teaching materials, creating powerful new immersive environment through which learners can acquire knowledge and develop higher level thinking skills. However, since the related 3D production tools are complicated and require users of professional trainings, applying 3D animation techniques in usual teaching activities still remains challenging.

Mennell Media develops an interactive 3D animation platform and designs several prototypes for Literacy Education, Foreign Language Education and Science Education [7]. The basic idea is to combine 3D animation content with effective sound designs, particularly music, and occasional visual mayhem. Based on the 3D interactive animation techniques and effective sound design, one can devise narratives that work within the limits of teaching needs. These techniques can greatly inspire young students' motivation and thirst for knowledge.

ISN Virtual Worlds [8] is another software company who applies web 3D and virtual worlds technologies for business and $3 \mathrm{D}$ training simulations and has developed a qualified experience in managing complex projects of web 3D, virtual worlds, 3D learning simulations and 3D interactive platforms.

However, from their provided demos, it is not available to reach the higher level of realistic feeling and immersiveness. $3 \mathrm{D}$ animation content should offer new perspectives through which the pedagogical principles can be touched by bringing the user closer to the real life experience and by emphasizing aspects. In each of these virtual rooms the user is shown a scene with various objects that he/she can interact according to the laws of physics. The architecture of the system is as follows:

- Sensors: read user's movements. At least these sensors can be the keyboard and the mouse;

- Drivers: allow the sensors to interact with the content;

- Physics engine: performs calculations and establishes the behavior of various components present in the scene according to user's interactions;

- 3D display: create a higher level of virtual reality. Moreover, such 3D interactive system is expected to have,

- Determining level of interaction of the subject with the 3D content, that is,

- Basic demonstrative level with 3D expositive presentation: the main interaction remains in $2 \mathrm{D}$ and only some representations are in $3 \mathrm{D}$;

- 3D mouse based interaction with limited access to the virtual world;
- Virtual reality level: real 3D interaction using dedicated sensors and dedicated 3D outputs for realistic feeling at the user level;

- Determining the emotional impact and its relationship with the subject;

- Determining the physical impact on the users;

- The strategy of combining 2D with 3D content where full $3 \mathrm{D}$ representation might not have enough impact or it might be very difficult to build;

- Synchronizing real time interaction with voice, chat and video. For distance learning, this gives learners the experience of collaborative learning within the interactive learning system.

\section{ANIMATION TRENDS IN TEACHING}

The purpose of this section is to present the potential trends of 3D animation content in the classroom based on available hardware architectures. Hence, the interactivity will be mainly through keyboard and mouse while the output will be displayed on conventional screens. Extensions, such as 3D goggles and specialized sensors (that read user's motions) will be mentioned but they will not be regarded as the emphasis of this section. These expensive devices will be experimental enhancements. It should be aware of the fact that using advanced and expensive hardware limits the potential users who are not ready to access to such expensive hardware devices. Therefore, we present a conceptual description of hardware-software design for developing such $3 \mathrm{D}$ interactive prototype system here.

\section{A. Sensor Description}

Nowadays, a lot of sensors can be used to read user movements and actions. However, due to practical limitations the developing prototype system will deploy $3 \mathrm{D}$ content controlled through traditional keyboard and mouse. For the higher level of interaction, the users can interact with the content by using an array of specialized sensors, e.g. haptic devices.

\section{B. Drivers}

The system architecture requests to connect the sensors to the central processing unit as well as to connect various display peripherals (3D screens, 3D goggles). This should be viewed as a custom designed architecture for best performance.

\section{Physics Engine}

Physics engine is the kernel of the entire system that takes as inputs signals from sensors, calculates trajectories of bodies according to their properties and the given inputs and then sends the output to the displays (3D screens, 3D goggles etc.). The physics engine uses advanced numerical modeling methods in order to simulate the physics as accurately as possible. Additionally, physically based rendering techniques [9] will be applied for realistic feeling as well.

\section{3D Display Device}

As an output device the desired prototype system will make use of a conventional display device. This is due to the cost and the need to make such 3D interactive animation system work well on the existing devices. However, for the 
higher level of immersiveness, the system will also consider the possibility to deploy the content on specialized output devices. For example,

- 3D screen. Toshiba, Sony etc. have some relevant products in this field;

- 3D goggles. Users wear the goggles and visualize the entire 3D scene.

Most of the 3D display technologies operate on a single basic principle - tricking our brain into interpreting 2D images into one with depth. This is usually fulfilled in the most basic way, i.e. stereoscopy, in which two eyes separately see two images with optical parallax so that the brain merges together into a 3D image. Stereoscopic images can be produced through a variety of different methods. The critical point here is the stereoscopic image format that is the foundation of image display, storage, and communication. There are two convenient formats to store stereoscopic images on internet, PNS and JPS. Essentially, the file is a standard JPG file that contains a stereo pair in a cross-eyed format.

\section{E. Anaglyph Images}

Anaglyph images are used to provide a stereoscopic 3D effect through a special glass. In essential, each lenses of the glass are of filters of different (usually chromatically opposite) colors, such as red and cyan. 3D images are made up of these two filtered colored layers, superimposed, but offset with respect to each other to produce depth effect. Usually the main subject is in the center, while the foreground and background are shifted laterally in opposite directions. The distinct advantage of the anaglyph 3D images is the cheap glasses. Low cost paper frames and plastic-framed glasses with accurate color filters are available everywhere. It has been applied to the images and video on internet and Blu-ray Discs.

\section{F. Polarization Systems}

This system is made up of a pair of polarizing filters that are orthogonal to each other (typically at 45 and 135 degrees). To present a stereoscopic motion picture, a scene is projected separately through different polarizing filters and superimposed onto the same screen. The viewer wears low-cost eyeglasses which also contain a pair of polarizing filters oriented differently (clockwise/counterclockwise with circular polarization or at 90 degree angles, usually 45 and 135 degrees, with linear polarization). As each filter passes only that light which is similarly polarized and blocks the light polarized differently, each eye sees a different polarized image. 3D effect can be generated by projecting these two polarized images separately into both eyes and depicting from slightly different perspectives accordingly. Since no head tracking is involved, the entire audience can view the stereoscopic images at the same time.

\section{G. The Pullfrich Effect}

Pullfrich 3D glasses have been applied to dynamic stereoscopy or 3-D visualization in visual reality. The basic principle is that the brain's response to the visual information from eyes at low light levels is slower. When selectively limiting the light level to one eye, the relative delay to perceive images can generate an illusion of depth. Occluding one eye and moving an image from right to left (or left to right, but NOT up and down), it will appear to move in depth, either toward or away from the viewer. Obviously, such glasses depend on motion. This will be invalid for the stationary objects.

\section{H. Eclipse Method}

Eclipse method usually adopts a mechanical shutter that blocks light from each appropriate eye when the converse eye's image is projected on the screen. The projector alternates between left and right images, while the shutters in the glasses or viewer are turned on or off in synchronization with the images on the screen. A variation on the eclipse method is used in LCD shutter glasses. Such glasses containing liquid crystal that will pass light through when the switch is on in synchronization with the images on the cinema, TV or computer screen. To sync with the projectors, the glasses are always connected to the projectors through wires.

\section{Volumetric Display}

Volumetric 3D display is defined as a visual representation of an object in 3D space in contrast to the traditional stereo display that simulates depth based on planar image pairs. Some devices have been developed for some special applications, such as, Lenticular-sheet display, swept-volume display, and fog display. However, such technologies still remain under investigation. In computer field, Human-Computer Interfaces (HCI) focuses on the volumetric display as well. The concerning properties may include, 360-dgree viewing, agreement of converge and accommodation cues, and the inherent 3-dimensionality, in particular, new user interface techniques. For example, the touch screen is widely utilized in tablet, mobile phones and ATM. Thus, next-generation of 3D display techniques will have to take into account user interface.

\section{CONCLUSION}

As a complementary learning approach, animation always stimulates students' interest in learning. The key point is to integrate the animation content into the teaching activities in the classroom. Realistic feeling, immersiveness and interactivity are always regarded as the criteria of choosing animation content in teaching activities.

This survey focuses on hardware advances and presents some potential trends of animation content in the classroom accordingly. Hardware environment is still the central issue of computer animation. In future, we will also study the pedagogical issues based on the latest 3D interactive animation content.

\section{REFERENCES}

[1] Go Animate. [Online]. Available: http://www.goanimate.com/

[2] Storytelling. [Online]. Available: http://www.xtranormal.com/

[3] M. Hegarty, "Dynamic visualizations and learning: getting to the difficult questions," Learning and Instruction, vol. 14, pp. 343-351, 2004.

[4] S. K. Card, J. D. Mackinlay, and B. Schneiderman, Readings in information visualization: Using vision to think, San Diego, CA: Academic Press, 1999.

[5] R. Spence, Information visualization, Essex: ACM Press, 2001. 
[6] Animasher. [Online]. Available: http://www.animasher.com/

[7] Mennell Media. [Online]. Available: http://www.mennellmedia.co.uk/

[8] Look Heaven and Earth. [Online]. Available: http://www.isnvirtualworlds.com/

[9] M. Pharr and G. Humphreys, Physically Based Rendering: From Theory to Implementation, Morgan Kaufmann Publishers Inc., 2004.

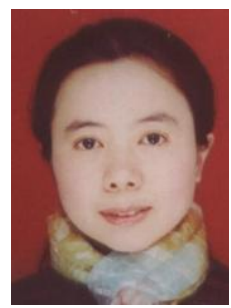

Lirong Xiao received the M.A. degree in English education from the Foreign Language Institute of Sichuan Normal University, Chengdu city, China, in 2006. She was an English teacher in Foreign Language middle school, Chengdu city, China. She is currently an English teacher in Guangzhou Overseas Chinese Foreign Language School, Guangzhou city, China. Her interests include, English teaching from primary to adult levels and multimedia teaching. 